\title{
Gesichtsmasken im Unterricht
}

\section{Vor- und Nachteile der Bedeckung der unteren Gesichtshälfte in Zeiten der Corona-Pandemie}

\author{
Korrespondenzadresse \\ Prof. Dr. Dr. Manfred Spitzer \\ Universität Ulm \\ Abteilung für Psychiatrie \\ Leimgrubenweg 12-14 \\ 87054 Ulm
}

\author{
Bibliografie \\ DOI https://doi.org/10.1055/a-1162-5343 \\ Nervenheilkunde 2020; 39: 522-532 \\ (c) Georg Thieme Verlag KG Stuttgart · New York \\ ISSN 0722-1541
}

Zusammenfassung: Neben dem Einhalten von körperlichem Abstand gehört das Tragen von Gesichtsmasken zu den wirksamsten und am besten untersuchten Verfahren, um die Verbreitung des Virus SARS-CoV-2 zu verhindern, insbesondere deshalb, weil diese Verbreitung durch asymptomatische Personen erfolgen kann, wie dies bei der derzeitigen Covid-19-Pandemie der Fall ist. Das Bedecken der unteren Hälfte des Gesichts mit einer Maske reduziert jedoch die unbewusst ablaufenden Fähigkeiten, zu kommunizieren und den Gesichtsausdruck anderer zu interpretieren und nachzuahmen. Positive Emotionen können überschattet und negative Emotionen verstärkt werden, wenn man den Mund des anderen nicht sieht. Auch die für den Menschen typische emotionale Mimikry (d. h. das wechselseitige Imitieren der Emotionen des jeweils anderen), die (auch dadurch erfolgende) emotionale Ansteckung und Emotionalität im Allgemeinen werden reduziert und damit letztlich die zwieschenmenschliche Bindung, z. B. zwischen Lehrenden und Lernenden. Da Emotionen eine wichtige Triebfeder für den Gruppenzusammenhalt sind, kann die verminderte Emotionalität, insbesondere die verminderte positive Emotionalität, das reibungslose Geschehen im Klassenzimmer beeinträchtigen. Angesichts der Tatsache, dass Lernprozesse durch Emotionen bekanntermaßen beschleunigt oder überhaupt erst ermöglicht werden, ist es wahrscheinlich, dass Gesichtsmasken zu einer gewissen Beeinträchtigung des Lernerfolges führen können. Das Für und Wider von Gesichtsmasken in Schulen sollte daher sehr ernsthaft und gründlich erwogen und von Lehrern und Schülern diskutiert werden. Die für den Unterricht Verantwortlichen sollten die Vor- und Nachteile in der spezifischen Situation der Schule abwägen und eine informierte Entscheidung im Hinblick auf die Anwendung von Gesichtsmasken im Unterricht treffen.

\section{Einleitung}

Das neue Coronavirus SARS-CoV-2 hat eine globale Pandemie der Krankheit Covid-19 verursacht, mit - Stand 31. Juli 2020 - fast 300000 Neuinfektionen weltweit an einem Tag, mehr als 17 Millionen bestätigten Infektionen insgesamt und mehr als 670000 Todesfällen [17]. Ursprünglich als eine Form der Grippe mit Fieber und Husten betrachtet hat sich die Krankheit als weitaus schwerwiegen- der erwiesen, da sie nicht nur die Lunge, sondern auch Leber, Herz, Nieren und Gehirn, mit Symptomen wie Anosmie [13] und Denkstörungen aufgrund von Defekten der neuroaxonalen Integrität (selbst bei leichten und mittelschweren Fällen) [1]. Bei Schwerkranken bewirkt das Virus einen zweigleisigen Angriff durch Störungen des Immunsystems (Zytokinsturm) als auch des Gerinnungssystems (Multiorgan-Thrombose) $[33,68]$, wodurch es zu akuten Gehirninfarkten mit chronischen neurologischen Defiziten kommen kann [62].

Im Vergleich zu Erwachsenen erkranken Kinder seltener, und wenn, dann verläuft ihre Erkrankung meist milde [34]. Um die Ausbreitung des Virus einzudämmen, wurden im März 2020 zusammen mit anderen Maßnahmen der physischen Distanzierung und des wirtschaftlichen Lockdowns Schulschließungen implementiert, von denen weltweit mehr als 1,5 Milliarden Schüler betroffen waren oder noch immer betroffen sind [74]. Diese Schließungen dauerten nur wenige Wochen (Dänemark) bis zu mehreren Monaten (Italien und vielen anderen Ländern) [16] und führten zu deutlich verminderten Bildungszuwächsen [77], zu Hunger (weil Schulspeisungen nicht mehr erfolgten), zu einer Zunahme des Kindesmissbrauchs (weil die Kinder nicht mehr vom Schulpersonal beobachtet wurden) und zu dem Risiko, „die Lebenschancen einer ganzen Generation junger Menschen zu beeinträchtigen“ (wie mehr als 1500 Unterzeichner eines offenen Briefs der Vereinigung britischer Kinderärzte formulierten) [16] aufgrund der langfristigen psychologischen, physiologischen, erzieherischen und wirtschaftlichen Belastung, welche die Gesellschaften ihren schwächsten Mitgliedern aufbürden.

Solange es keinen Impfstoff und keine spezifische medikamentöse Behandlung gibt, müssen wir die erste Pandemie des 21. Jahrhunderts mit Methoden aus dem 14. bis 19. Jahrhundert bekämpfen: Distanzierung, Händewaschen und Abdecken von Mund und Nase mit einem Tuch. Tatsächlich sind Gesichtsmasken zu einem immer wichtigeren Teil der nationalen Strategien zur Bekämpfung der Corona-Pandemie geworden. Angesichts der Tatsache, dass Schulschließungen bereits beendet sind oder irgendwann beendet werden müssen, stellt sich damit die Frage des Tragens von Gesichtsmasken an Schulen während der nächsten Phase der Pandemie, in der die Zahl der neuen Fälle zurückgeht, das Virus aber 
immer noch unter uns weilt. Dies ist aus den folgenden Gründen besonders dringlich:

- Menschen ohne jegliche Symptome können infiziert sein und das SARS-CoV-2-Virus unbemerkt verbreiten.

- Beim Sprechen wird das Virus in die Luft freigesetzt, je lauter, desto mehr.

- In Klassenzimmern kann das Virus über viele Stunden in der Luft bleiben.

- Das Tragen von Masken ist vergleichsweise billig sowie einfach umzusetzen und zu überwachen.

Diesen Gründen für das Tragen von Masken stehen die folgenden Nachteile entgegen:

- Das Tragen von Gesichtsmasken kann körperliche Nebenwirkungen hervorrufen.

- Gesichtsmasken beeinträchtigen die Gesichtserkennung und Gesichtsidentifikation.

- Gesichtsmasken beeinträchtigen die verbale und nonverbale Kommunikation.

- Gesichtsmasken blockieren die meist unbewusst ablaufende emotionale Kommunikation zwischen Lehrer und Schüler.

Angesichts dieser Vor- und Nachteile ist es nicht klar, ob Gesichtsmasken in Zeiten der aktuellen Viruspandemie im Bildungsbereich eingesetzt werden sollten. Darüber hinaus können angesichts der Vielfalt der Bildungseinrichtungen in Bezug auf Land, Kultur und Alter der Lernenden verschiedene Aspekte unterschiedlich gewichtet werden. Weitere Aspekte der Pandemie, die Zahl der Infizierten, die Ausbreitungsgeschwindigkeit und die lokalen Gegebenheiten der Infektionsfälle, können die Entscheidung bezüglich der Verwendung von Gesichtsmasken im Klassenzimmer ebenfalls beeinflussen. Die Frage sollte daher dringend und anhand des vorhandenen Wissens über die Auswirkungen von Gesichtsmasken diskutiert werden, da sie weltweit mehr als 2 Milliarden Schüler, Lehrer und Schulpersonal direkt betrifft sowie darüber hinaus indirekt auch deren Familien.

\section{Gesichtsmasken vermindern die Infektions- wahrscheinlichkeit deutlich}

Diese Pandemie war ein bewegliches Ziel, was unser Wissen über sie betrifft. ${ }^{1}$ Die Frage des Tragens von Gesichtsmasken kann als Paradebeispiel dienen. Während der Zeit der Schulschließungen (etwa von Mitte März bis April und/oder Mai 2020) wurde deutlich, dass Gesichtsmasken, die Mund und Nase in geschlossenen öffentlichen Räumen (öffentliche Verkehrsmittel, Geschäfte, Restaurants usw.)

1 Dies war vor 100 Jahren auch nicht anders, nachdem der französische Bakteriologe Charles Nicolle im Oktober 1918 - also auf dem Höhepunkt der damaligen Pandemie - herausgefunden hatte, dass das Agens, welches Influenza verursacht, deutlich kleiner sein muss als alle bekannten Bakterien. Die führte sogar in den Zeitungen US-amerikanischer Kleinstädte zu Spott gegenüber dem Tragen von Gesichtsmasken, etwa mit Aussagen wie der, dass das Tragen von Gesichtsmasken gegen Influenza etwa so sinnvoll sei wie „die Verwendung von Stacheldraht als Schutz gegen Fliegen “ [15]. Dies brachte die Leute eher von der Benutzung von Gesichtsmasken ab und führte zu vermehrtem Misstrauen in der Allgemeinbevölkerung. bedecken, ein wirksames Mittel sind, um die Ausbreitung des Virus und damit die Verschlimmerung der Pandemie zu verhindern. Dies war zum Zeitpunkt des Ausbruchs der Pandemie in den meisten Ländern (Februar 2020) noch nicht klar gewesen, da Institutionen wie die Weltgesundheitsorganisation (WHO), die US-amerikanischen Centers of Disease Control and Prevention (CDC) und z. B. das deutsche Robert-Koch-Institut (RKI) sowie viele Regierungen auf der ganzen Welt die Verwendung von Gesichtsmasken zur Bekämpfung von Covid-19 nicht empfahlen. ${ }^{2}$

Mit der Wiedereröffnung der Schulen im April und Mai änderte sich diese Situation [2, 26, 45] und im Juni 2020 gab es keinen Zweifel mehr ( $\triangleright$ Abb. 1), dass das Tragen von Gesichtsmasken eine der wirksamsten Präventionsmaßnahmen ist, die Menschen ergreifen können, um sich und andere vor einer Infektion mit dem Virus zu schützen [67]. Da das Tragen von Masken durch infizierte Personen das Übertragungsrisiko verringert und wegen des hohen Anteils asymptomatischer Infizierter und Übertragungen gibt es inzwischen gute Gründe für die Wirksamkeit einer weit verbreiteten Verwendung von Gesichtsmasken zur Verringerung der Verbreitung von COVID-19. Kurz gesagt, da es klar ist, dass Menschen infiziert und ansteckend sein können, auch wenn sie noch keine Symptome von Covid-19 zeigen - was eine Besonderheit des SARS-CoV-2-Virus ist - können Gesichtsmasken die Auswirkungen dieses besonders gefährlichen Virus definitiv mildern.

So wurde in einer chinesischen Studie aus Wuhan geschätzt, dass bis zu 79\% der Virusinfektionen von undiagnostizierten, vermutlich asymptomatischen Personen verursacht wurden [47]. In 2 weiteren Studien, eine aus 2 Umfragen unter 2812 und 2343 Einwohnern der Kleinstadt Vó in Italien und eine weitere aus 1032 Beschäftigten des Gesundheitswesens (einschließlich des patientenseitigen Frontlinienpersonals wie Ärzte, Krankenschwestern und Physiotherapeuten) im Vereinigten Königreich, wurden ähnliche Ergebnisse berichtet [42]. In der italienischen Studie waren 42,5\% der bestätigten SARS-CoV-2-Infektionen asymptomatisch, und in der britischen Studie wiesen 60 \% keine oder nur sehr leichte Symptome auf. Das heißt, man erkrankt durch den Kontakt mit Menschen, die scheinbar gesund sind. Aus diesem Grund ist es bei der gegenwärtigen Covid-19-Pandemie nicht möglich, dass nur die Menschen Masken tragen, die ansteckend sind. Jeder kann infektiös sein, ohne es zu wissen [42]. Die einzige Möglichkeit, Infektionen zu verhindern, besteht daher darin, dass alle Menschen Gesichtsmasken tragen.

Seit mehr als 70 Jahren ist bekannt, dass beim Sprechen orale Flüssigkeitströpfchen entstehen, deren Größe stark variiert, von deutlich sichtbaren bis zu unsichtbar kleinen Aerosolen [24]. Je lauter jemand spricht, desto mehr Tröpfchen und Aerosole werden erzeugt. Unabhängig von ihrer Größe können sie alle infektiöse Viruspartikel beherbergen und Viruserkrankungen verbreiten [2, 5,

2 Der Grund für diese Fehlinformation wurde mit der Zeit wirklich klar: Es gab ganz einfach zu wenig Masken. Man wollte sicherstellen, dass die wenigen vorhandenen Masken auch von denjenigen verwendet werden können, die sie am nötigsten brauchen, und gab die vorhandenen Masken daher und andere Schutzkleidung an Krankenhäuser ab, die nur wenige Vorräte hatten. Es wäre sicher besser gewesen, die Verantwortlichen hätten dies genau so kommuniziert und den Leuten gesagt, dass sie ihre eigenen Gesichtsmasken nähen (und diese sogar verkaufen) können. 

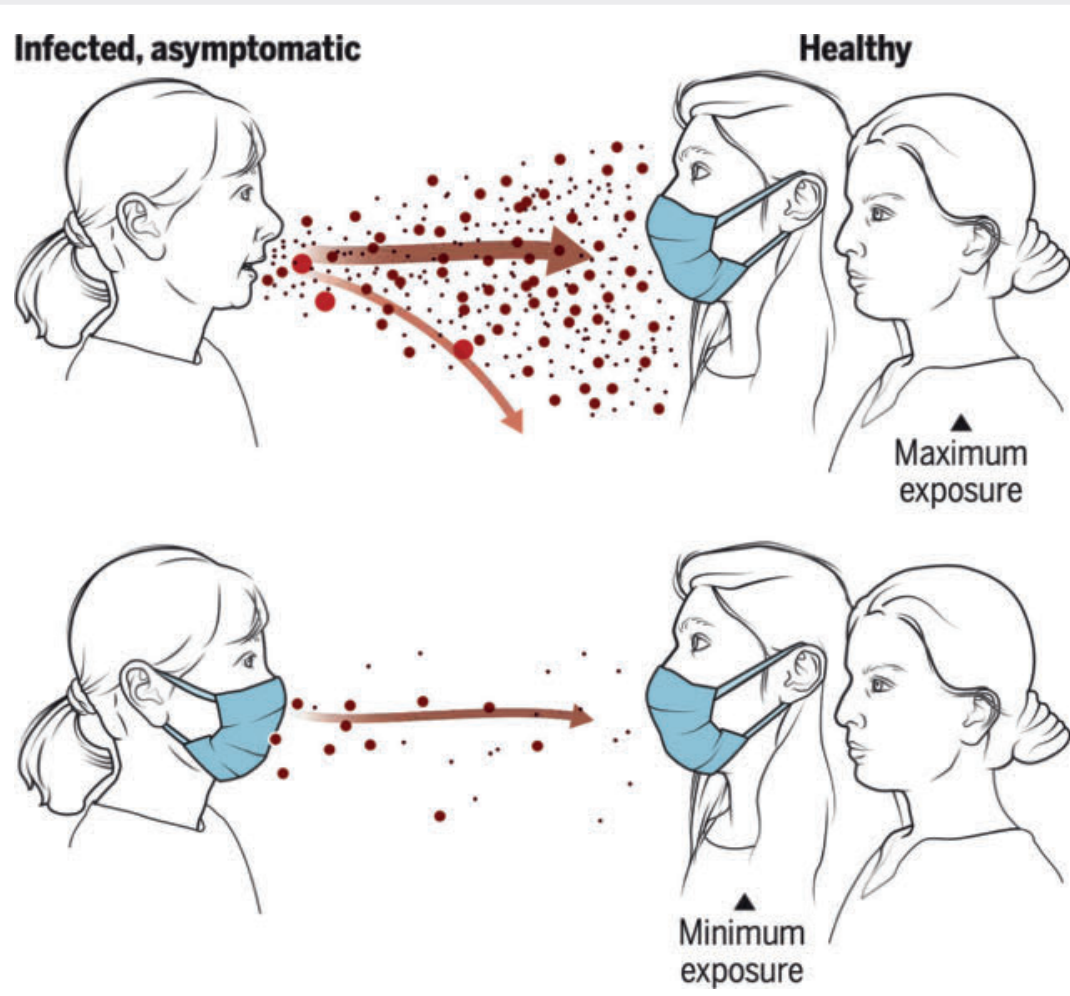

- Abb.1 Gesichtsmasken reduzieren die Übertragung des SARS-CoV-2-Virus über die Luft. Die Autoren kommentieren: „Infektiöse Aerosolpartikel können von asymptomatisch infizierten Personen beim Atmen und Sprechen freigesetzt werden. Werden keine Masken getragen, ist das Risiko einer Infektion am größten, trägt jeder eine Maske, ist es am kleinsten“. Abdruck mit freundlicher Genehmigung des Verlages [67]

6]. Tatsächlich hat sich gezeigt, dass das Virus über viele Stunden in der Luft in einem geschlossenen Innenraum wie einem Klassenzimmer übertragen wird [46]. Wie im Mai 2020 in der Zeitschrift Science online veröffentlicht wurde: „[...] Berechnungen sagen voraus, dass sich in ruhiger Luft ein 100- $\mu \mathrm{m}$-Tröpfchen in 4,6s aus 8 Fuß Höhe auf dem Boden absetzen wird, während ein 1- $\mu$ m-Aerosolpartikel 12,4 Stunden [zum Absetzen] benötigt. Messungen zeigen nun, dass intensives Husten und Niesen, das größere Tröpfchen über 20 Fuß hinaus treibt, ebenfalls Tausende von Aerosolen erzeugen kann, die sich noch weiter fortbewegen können. [...] unter vielen Innenraumbedingungen, unter denen Aerosole stundenlang in der Luft bleiben, sich mit der Zeit ansammeln und Luftströmungen über Entfernungen von mehr als 6 Fuß folgen können “ [47]. Die Autoren fassen ihre Perspektive mit einer sehr überzeugenden Zeichnung zusammen, die in $>$ Abb. 1 wiedergegeben ist.

Es gibt zudem epidemiologische Daten zur Wirksamkeit des Tragens von Gesichtsmasken gegen die Verbreitung des neuen Coronavirus: In Hongkong benutzten $96 \%$ der Bevölkerung einen Mundschutz, und die Inzidenz der neuen Fälle lag im März bei 129 pro Million Einwohner, während in Spanien, Italien und Deutschland, wo zu dieser Zeit keine Mundschutz getragen wurde, die Inzidenz 2983 (Italien), 2251 (Spanien) und 1242 (Deutschland) pro Million Einwohner betrug [31]. Kurz nachdem der Bürgermeister der deutschen Stadt Jena (mit etwa 110000 Einwohnern) am 6. April eine Anordnung zur Verwendung von Gesichtsmasken für die gesamte Stadt zur Bekämpfung der Ausbreitung des Virus eingeführt hatte (3 Wochen früher als im restlichen Deutschland), gab es in dieser Stadt an 9 aufeinander folgenden Tagen keinen einzigen neuen Fall. Verglichen mit einem „Surrogat“-Jena (ein gewichteter Mix aus 4 deutschen Städten) als Kontrolle, betrug dieser Rückgang an neuen Fällen $23 \%$ [56].

Ein am 16. Juni 2020 veröffentlichtes naturalistisches Experiment verwendete die unterschiedlichen Zeitpunkte (vom 8. April bis 15. Mai), an denen 15 US-Bundesstaaten die Verwendung von Gesichtsmasken im öffentlichen Raum zur Pflicht machten, um die Verbreitung von Covid-19 einzudämmen [52]. Die Studie ergab, dass die obligatorische Verwendung von Gesichtsmasken mit einem Rückgang der täglichen Covid-19-Wachstumsrate um $2 \%$ in den 2-3 Wochen danach einherging. Auf den ersten Blick mag dieser Effekt klein erscheinen, aber ein kumulierter Rückgang um $2 \%$ täglich über 3 Wochen bedeutet einen Rückgang der Infektionsraten über den Gesamtzeitraum von über $40 \%$. Dementsprechend schätzten die Autoren anhand ihrer Daten, dass bis zum 22. Mai 2020 durch die Verwendung von Gesichtsmasken bereits 230000 bis 450000 neue Fälle von Covid-19 verhindert werden konnten.

Schließlich wurden in einer großen, umfassenden Übersicht und Metaanalyse, die am 1. Juni 2020 im medizinischen Fachblatt Lancet online veröffentlicht wurde, Daten aus 172 Beobachtungsstudien aus 16 Ländern verwendet, darunter 44 vergleichende Studien [14]. Das Ergebnis war beeindruckend und klar: Gesichtsmasken (chirurgischer Typ) reduzierten das Infektionsrisiko um 85\%. Da Gesichtsmasken vergleichsweise billig und einfach zu verwenden sind, gehören sie zu den einfachsten und unkompliziertesten Mitteln, um die Übertragung des neuen Coronavirus einzudämmen [53]. 


\section{Die Nebenwirkungen von Gesichtsmasken}

Es ist eine medizinische Binsenweisheit, dass alles was wirkt, auch Nebenwirkungen hat. Über die körperlichen Nebenwirkungen von Gesichtsmasken haben vor allem Mediziner und Wissenschaftler, die in der Chirurgie sowie in Labors arbeiten, berichtet. In einer Studie mit 158 Beschäftigten im Gesundheitswesen während der Covid-19-Pandemie war die häufigste Nebenwirkung bei längerem Tragen von Gesichtsmasken beidseitige Kopfschmerzen, über die etwa $80 \%$ der Teilnehmer berichteten [60]. Die Kopfschmerzen traten 1- bis 4-mal während eines 30-tägigen Zeitraums der Maskenanwendung auf und wurden von der Mehrheit (72\%) der Befragten als leicht eingestuft. Innerhalb von 30 Minuten nach Abnahme der Gesichtsmaske lösten sich die Schmerzen spontan auf. Schmerzmedikamente (z. B. nicht steroidale Analgetika) wurden nur in wenigen Fällen eingesetzt.

Gesichtsmasken können ferner eine periorale Dermatitis mit Ausschlägen und Rötungen durch Speichel, Schweiß und Feuchtigkeit zwischen Maske und Haut verursachen. Dies kann ein Nährboden für Bakterien sein. Juckende Ausschläge können durch eine irritative Dermatitis verursacht werden, die durch die Maske und/ oder die daran befestigten Gummischnüre verursacht wird, die an der Haut von Nase und Ohren reiben [65].

Eine experimentelle Studie über die physischen Auswirkungen des Tragens entweder einer chirurgischen oder einer N95-Gesichtsmaske wurde mit je 5 gesunden männlichen und weiblichen Versuchspersonen durchgeführt, die auf einem Laufband in einer Klimakammer bei $25^{\circ} \mathrm{C}$ Lufttemperatur und einer relativen Luftfeuchtigkeit von $70 \%$ trainierten, während sie Gesichtsmasken trugen. Beide Arten von Gesichtsmasken führten zu signifikanten Temperatur- und Feuchtigkeitsunterschieden im Mikroklima zwischen Gesichtsmaske und Haut. Insbesondere führte die N95-Maske (entspricht einer FFP-2-Maske) im Vergleich zur chirurgischen Maske zu einer höheren Feuchtigkeit und Hauttemperatur im Bereich zwischen Gesichtsmaske und Haut, zu einer höheren Herzfrequenz und zu einer Zunahme negativer „Empfindungen, einschließlich des Gefühls der Abgeschlagenheit, Enge, Juckreiz, [und] Müdigkeit“" [46]. Eine Studie zu den kardiovaskulären Auswirkungen von Gesichtsmasken (chirurgisch oder FFP-2) auf die Leistungsfähigkeit gesunder junger männlicher Probanden auf dem Fahrradergometer ergab, dass die körperliche Leistungsfähigkeit durch chirurgische Masken um gut $2 \%$ (nicht signifikant) und durch FFP-2-Masken um etwa $5 \%(p<0,01)$ reduziert war [32]. Insgesamt bevorzugten in beiden Studien die Probanden die chirurgische Gesichtsmaske. Dieser Befund führte unter anderem dazu, dass N95-Masken nie der Allgemeinheit zum Tragen empfohlen wurden, da sie nicht nur teurer sind, sondern auch - wegen des damit verbundenen Unbehagens - mit weitaus geringerer Wahrscheinlichkeit von Jedermann über längere Zeiträume getragen werden. Selbst Chirurgen verwenden sie nicht.

Im Hinblick auf die Prävention von Covid-19 wurden weiterhin die zusätzlichen Bedenken hinsichtlich des Tragens von Gesichtsmasken geäußert:

- Das Tragen einer Gesichtsmaske kann ein falsches Gefühl der Sicherheit vermitteln und dazu führen, dass sich die Menschen weniger an andere Maßnahmen halten, die zur Infektionskontrolle wie beispielsweise Abstand halten.
- Aufgrund von Problemen mit dem Sprachverständnis können Menschen ohne Absicht beim Gespräch näher zusammenrücken und dadurch das Infektionsrisiko erhöhen.

- Das Tragen von Gesichtsmasken kann dazu führen, dass Brillengläser beschlagen, was Folgen haben kann, die von leichtem Unbehagen bis hin zu schweren Unfällen reichen können.

- Das Tragen einer Gesichtsmaske führt dazu, dass die ausgeatmete Luft in die Augen gelangt. „Dies erzeugt ein unangenehmes Gefühl und den Impuls, sich die Augen zu reiben. Wenn die Hände mit Viren kontaminiert sind, infiziert man sich dann selbst“, schreiben hierzu Lazzarino und Mitarbeiter im Fachblatt British Medical Journal [44].

- Darüber hinaus wird bei jedem Atemzyklus ein Teil des zuvor ausgeatmeten Kohlendioxids wieder eingeatmet. Dies erhöht sowohl die Atemfrequenz als auch die Atemtiefe. Dadurch kann sich die Viruslast in der Umgebung erhöhen, wenn infizierte Personen, die Masken tragen, rascher und tiefer atmen und damit mehr kontaminierte Luft produzieren und verbreiten. „Dies kann auch den klinischen Zustand der Infizierten verschlechtern, wenn die vermehrte Atmung mehr Viren in die tieferen Teile der Lungen transportiert“, fügen die Autoren hinzu [44].

- Wenn infizierte Menschen die Luft in der Maske wieder einatmen, steigt ihre Viruslast an, d. h. die Menge an Viren, denen sie dauernd ausgesetzt sind. Auch dies könnte sich negativ auf den Krankheitsverlauf auswirken.

Zusammenfassend kann man festhalten: Gesichtsmasken verringern zwar prinzipiell die Ausbreitung von Viren während der Pandemie, können sie aber unter bestimmten Bedingungen auch verschlimmern. Zusätzlich zu diesen Problemen der körperlichen Gesundheit müssen 3 weitere Probleme bei der Verwendung von Gesichtsmasken betrachtet werden: Die Beeinträchtigung von Gesichtserkennung und -identifikation, Beeinträchtigung der Kommunikation sowie die Blockierung emotionaler Signale von der unteren Gesichtshälfte.

\section{Gesichtsmasken beeinträchtigen Gesichts- erkennung und Gesichtsidentifizierung}

Im täglichen Leben sind menschliche Beobachter äußerst geschickt darin, Gesichter zu erkennen, zwischen ihnen zu unterscheiden und daraus eine Vielzahl von Informationen über den Betreffenden abzuleiten: Zum einen statische Merkmale wie Alter, Geschlecht oder Identität und zum anderen veränderliche Merkmale wie Blickrichtung, Lippenbewegungen oder emotionale Zustände.

Der Mensch ist biologisch programmiert, Gesichter zu erkennen. Schon Neugeborene zeigen eine Vorliebe dafür, menschliche Gesichter zu betrachten, selbst rudimentäre Zeichnung von Gesichtern, die man ihnen zeigt. Im Alter von 7 Monaten sind Säuglinge bereits in der Lage, wütende oder ängstliche Gesichter zu erkennen [64]. Die Bevorzugung von Gesichtern bei der Wahrnehmung gegenüber anderen visuell dargebotenen Reizen nimmt im zweiten Lebensjahr interessanterweise wieder ab [48].

Neben dieser angeborenen Präferenz für Gesichter haben soziale Erfahrungen in der Gemeinschaft eine zunehmende Bedeutung für das Wahrnehmen und Erkennen von Gesichtern. Zur Erforschung 
dieser Fähigkeiten bei Säuglingen werden mittlerweile so unterschiedliche Methoden wie Verhaltensanalysen, Analysen der Blickrichtung und der Augenbewegungen sowie neurowissenschaftliche Verfahren wie ereigniskorrelierte Potenziale (EKP), funktionelle Magnetresonanztomografie (fMRT) und Nahinfrarotspektroskopie (NIRS) verwendet. Hierdurch wurde eine verstärkte Reaktion von Verhalten bzw. neuronaler Aktivierung gegenüber

- der Mutter im Vergleich zu Fremden,

- weiblichen im Vergleich zu männlichen Personen,

- der eigenen Volksgruppe gegenüber anderen Volksgruppen und

- einheimischen gegenüber im Vergleich zu nicht einheimischen Gesichtern gefunden.

Darüber hinaus verändern sich

- die neuronalen Reaktionen von Säuglingen auf Gesichter bei der Kommunikation im Laufe der Entwicklung, was schließlich

- dazu führt, dass das sich entwickelnde Kind Gesichtern in besonderem Maße seine Aufmerksamkeit zuwendet, was sich in einer verbesserten Verarbeitung von und Reaktionsfähigkeit auf Gesichter zeigt, die in der heimischen Umgebung häufig anzutreffen sind.

Kurz: Wir erkennen und identifizieren die Gesichter unserer Altersgenossen schneller als Gesichter von Fremden [69], so wie wir die Laute (Phoneme) unserer Muttersprache besser erkennen als unbekannte Laute (Phoneme) aus unbekannten Sprachen [63].

Das Verbergen des unteren Teils des Gesichts mit einer Gesichtsmaske beeinträchtigt die Gesichtserkennung und Gesichtsidentifikation deutlich. Dies ist der Grund, warum Räuber sie tragen. Werden Gesichtsmasken zu einem Teil des normalen Alltags, wird allen Beteiligten immer deutlicher, in welchem Ausmaß das Verdecken des Gesichts unser tägliches soziales Miteinander sowie die Fähigkeit beeinträchtigt, andere Menschen zu erkennen. Im schulischen Umfeld wird die Fähigkeit, einander zu kennen und zu erkennen, normalerweise als selbstverständlich vorausgesetzt. Sie kommt daher überhaupt erst zu Bewusstsein - ähnlich wie die Atemluft -, wenn sie fehlt. Es gibt jedoch relativ einfache Lösungen für dieses Problem, wie z. B. deutlich sichtbare Namensschilder, „personalisierte“ Masken und unsere Fähigkeit, uns gegenseitig anhand unserer Stimme und unserer Körperform und -haltung zu erkennen und zu identifizieren. Im Gegensatz zum Problem der Erkennung und Identifizierung von Menschen ist das Problem der Kommunikation in Schulen mit Gesichtsmasken viel schwieriger zu lösen.

\section{Gesichtsmasken beeinträchtigen die verbale und nonverbale Kommunikation}

Eines der einfachsten und zugleich wichtigsten Werkzeuge in der menschlichen Kommunikation ist das Gesicht. Der Betrachter erkennt daran mühelos und sehr schnell Identität, Geschlecht, Geschlecht, Alter, ethnische Zugehörigkeit, sexuelle Orientierung, körperliche Gesundheit, Attraktivität, emotionalen Zustand, Persönlichkeitsmerkmale, Schmerz oder körperliche Lust, Täuschungsversuche und sogar den sozialen Status eines Menschen [38]. Wenn Fremde zusammentreffen, die 2 verschiedene Sprachen sprechen und sich gegenseitig daher nicht verstehen, können sie dennoch Gesichtsausdrücke wie Lächeln oder Stirnrunzeln mit Leichtigkeit interpretieren und sich so verständigen. Tatsächlich ist die grundle- gendste Form der Kommunikation zwischen Menschen die Mimik. Das liegt daran, dass unsere Gesichtsausdrücke eine einfache universelle Sprache darstellen, die wahrscheinlich biologisch bedingt ist und daher in allen Kulturen nahezu identisch ist. Der vergleichende Verhaltensforscher Irenäus Eibl-Eibesfeldt hat es zu seiner Lebensaufgabe gemacht, das weltweit größte Video-Archiv menschlicher Emotionsausdrücke zu erstellen. Man kann anhand vieler Beispiele zusehen, wie Menschen aus den verschiedensten Gegenden der Welt und mit den unterschiedlichsten kulturellen Hintergründen genau gleiche bewegte Gesichtsausdrücke produzieren, um ganz bestimmte Emotionen zu signalisieren [25]. Das mag der Grund dafür sein, dass viele Menschen das Tragen von Masken prinzipiell nicht mögen, weil es sie in ihren Möglichkeiten des Ausdrucks und der Wahrnehmung von Emotionen sehr stark beeinträchtigt.

Denn weil wir die untere Gesichtshälfte nicht sehen können, wenn jemand eine Maske trägt, ist unsere Fähigkeit, Menschen zu verstehen, eingeschränkt. Wir sind gezwungen, uns nur auf Sprache und Gestik zu verlassen, was die Interpretation von Nuancen einschränkt. Einzig die Augen sind über der Maske noch sichtbar und helfen dabei durchaus. Tatsächlich deuten Augenbewegungsstudien während des Betrachtens eines Gesichtes darauf hin, dass die Augen bei der Gesichtswahrnehmung zuerst und zudem am längsten beachtet werden und daher für die Kommunikation am aufschlussreichsten erscheinen. Sobald 2 Menschen beispielsweise ihren Blick auf etwas richten, kann dieses Etwas dadurch zum Mittelpunkt der gemeinsamen Aufmerksamkeit werden, was ein grundlegender Mechanismus ist, um Dinge gemeinsam zu untersuchen, Handlungen gemeinsam auszuführen und Probleme gemeinsam zu lösen.

Nach den Augen steht die Region von Mund und Nase an zweiter Stelle was unsere Aufmerksamkeit auf Gesichtspartien anbelangt. Für eine effektive verbale Kommunikation hat das Abdecken des Mundes 2 nachteilige Folgen: Erstens wird das Hörsignal beeinträchtigt, da Gesichtsmasken die Schallamplitude dämpfen und Frequenzbänder absorbieren können, die für das Verstehen gesprochener Sprache wichtig sind. Zweitens ist das Betrachten der Lippen vollständig blockiert. Obwohl es viele Menschen vielleicht noch nie bemerkt haben, wird dieses Signal von allen Menschen zur Unterstützung des Sprachverstehens verwendet. Von der Geburt bis zum Alter von etwa 8 Monaten schauen Babys auf den Mund ihrer Mutter, um den Schall in einzelne Sprachlaute (Phoneme) zu zerlegen und so die Muttersprache zu erlernen. Wenn sie 2-sprachig aufgezogen werden, müssen sie eine größere Anzahl von Phonemen lernen und fangen daher früher damit an, auf den Mund der mit ihnen sprechenden Mutter zu schauen und halten dieses Verhalten auch länger aufrecht, d. h. bis etwa zum Ende des 12. Lebensmonats [66]. Wenn wir erwachsen sind, neigen wir z. B. in lauten Umgebungen dazu, jemandem unter solchen Umständen beeinträchtigter Tonverständlichkeit, genau auf den Mund zu schauen. Gehörlose Menschen nutzen das Lippenlesen und verlassen sich dabei vollständig auf visuelle Hinweise, um Sprache zu verstehen (deshalb gibt es spezielle Gesichtsmasken mit einem transparenten Stück über dem Mund, um bei der Kommunikation mit gehörlosen Menschen deren Bedarf an visueller Spracheingabe zu decken).

Halten wir fest: Da die Sprachübertragung durch das Tragen von Masken beeinträchtigt wird, besteht die Gefahr von Missver- 
ständnissen, wenn Gesichtsmasken in Schulen weit verbreitet sind. Das Sprechen durch eine Gesichtsmaske kann höhere Frequenzen dämpfen und dadurch die verbale Kommunikation beeinträchtigen. Die Größe des Effekts hängt vom Sprecher, der Art der Maske, dem Gehör des Zuhörers und den Hintergrundgeräuschen ab und kann daher zwischen vernachlässigbar [54] und erheblich [8] variieren. Darüber hinaus ist bekannt, dass visuelle Hinweise bei der Spracherkennung helfen, was eine zusätzliche Ursache für die durch die Gesichtsmaske verursachte Beeinträchtigung der Sprachwahrnehmung und Kommunikation sein kann.

\section{Gesichtsmasken blockieren emotionale Signale zwischen Lehrenden und Lernenden}

Einzelne Merkmale des Gesichts wie Größe und Form der Nase, Farbe der Augen und ihre Konfiguration (die „Summe“ der „Teile“) werden nicht nur verwendet, um auf die Identität eines Menschen zu schließen, sondern auch auf dessen Emotion [11]. Kein anderer als Charles Darwin [19], der Begründer der Evolutionstheorie, war der erste, der die evolutionären Ursprünge des Gesichtsausdrucks von Emotionen untersucht hat. Selbst Kleinkinder unter einem Jahr sind in der Lage, Gesichtsausdrücke als soziale Signale zu verstehen, die die Gefühle anderer Menschen repräsentieren. Sie beginnen zunächst, auf ängstliche Gesichter zu reagieren, reagieren aber bald darauf auch auf fröhliche Gesichter. Emotionen spielen eine große Rolle in unseren sozialen Interaktionen, von denen Lehren und Lernen zu den kulturell relevantesten gehören. Man geht davon aus, dass die jüngste Entwicklung des menschlichen Gehirns durch seine erhöhte Fähigkeit zur kulturellen Weitergabe von Wissen, d. h. zum Lehren und Lernen, geprägt wurde [36].

Vor mehr als 50 Jahren führte der kalifornische Psychologe Paul Ekman, aufbauend auf Forschungen europäischer Ethologen aus der Schule von Konrad Lorenz, Experimente durch, um die Existenz von Grundemotionen im Gesichtsausdruck unabhängig von der Kultur nachzuweisen: Überraschung, Angst, Ekel, Wut, Glück und Traurigkeit [27, 28]. Es ist seit langem bekannt, dass negativer Affekt eine feinkörnigere perzeptive und kognitive Verarbeitung zur Folge hat, während positiver Affekt zu einer ganzheitlicheren Verarbeitung führt. Dementsprechend hat sich gezeigt, dass Emotionen, die in Gesichtern wahrgenommen werden, einen Einfluss darauf haben, wie Gesichter verarbeitet werden: Ein Gesicht, in dem eine negative Emotion wahrgenommen wird, wird weniger ganzheitlich verarbeitet als ein Gesicht, das eine positive Emotion zeigt [18]. Dies trifft auch auf andere Inhalte zu: Lächeln fördert die Kreativität [39], die umgekehrt unter Kritik und der damit verbundenen Angst reduziert ist. Blickt ein Schüler in das kritische Gesicht eines Lehrers, leidet die Kreativität, was zu dem bekannten und mittlerweile gut untersuchten Phänomen der Angst vor Mathematik führt: Diese bewirkt, dass man beim Lösen von Aufgaben weniger erfolgreich ist, was wiederum zu noch mehr Angst führt - unabhängig von der mathematischen Begabung [51].

Wie erwähnt, bietet das Gesicht eine universelle Sprache für die Kommunikation, insbesondere für die Kommunikation von Gefühlen. Die Mundregion eines Gesichts vermittelt Informationen, die für das Lächeln entscheidend sind, d. h. für eine positive Emotion, die als sozialer Klebstoff wirken kann und positives soziales Wahrnehmen und Handeln erleichtert [71]. Wenn man die untere Ge- sichtshälfte nicht sieht, ist es besonders schwierig, die positiven Emotionen eines Maskenträgers - Vergnügen, Freude, Glück, Belustigung, Geselligkeit und Freundlichkeit - zu erkennen, da sie im Wesentlichen durch ein Lächeln vermittelt werden. Daher beeinträchtigen Gesichtsmasken vor allem unsere positiven sozialen Interaktionen und unsere Fähigkeit, einander zu verstehen und uns ineinander einzufühlen. Zwar kann in einigen Kulturen ein Lächeln auch als Zeichen von Dummheit, Oberflächlichkeit und Unehrlichkeit gewertet werden, wie eine interkulturelle Studie über die soziale Wahrnehmung lächelnder Menschen gezeigt hat [41]. Dennoch besteht der weitaus überwiegender Teil der Funktionen des Lächelns in den meisten Gegenden der Welt in der Vermittlung positiver Emotionen.

Es gibt 2 Arten des Lächelns, das wahre Lächeln und das unehrliche Lächeln. Ein wahres oder echtes Lächeln, das in der wissenschaftlichen Literatur auch als Duchenne-Lächeln ${ }^{3}$ bezeichnet wird, betrifft sowohl den Mund (der Musculus zygomaticis major hebt die Mundwinkel an) als auch die Augen (der Musculus orbicularis oculi hebt die Wangen an, bildet Krähenfüße um die Augen und bewirkt, dass die Augenbrauen näher zusammenrücken, was senkrechte Falten, das sogenannte „Stirnrunzeln“, bewirkt). Im Gegensatz dazu beinhaltet das unehrliche Lächeln, das auch als „falsches Lächeln“, „Botox-Lächeln“, „Pan-Am-Lächeln“4 oder „Nicht-Duchhenne-Lächeln" bezeichnet wird, lediglich das willentliche Anheben der Mundwinkel, um bewusst Höflichkeit zu signalisieren. Eine Metaanalyse des Duchenne-Lächelns gegenüber dem Nicht-Duchenne-Lächeln ergab, dass sowohl das Duchenne-Lächeln als auch Menschen, die ein Duchenne-Lächeln erzeugen, positiver bewertet werden (d. h. authentisch, echt, wirklich, attraktiv, vertrauenswürdig) als das Nicht-Duchenne-Lächeln und Menschen, die ein Nicht-Duchenne-Lächeln erzeugen [35]. Außerdem war der Unterschied zwischen Duchenne-Lächeln und Nicht-Duchenne-Lächeln größer, wenn die Stimuli Videos (bewegte Gesichter) und nicht Fotos waren (d. h. wenn das Lächeln in lebendiger Bewegung zu sehen war) und wenn das Lächeln auf natürliche Weise und nicht durch Aufforderungen zum Lächeln (Posing) hervorgerufen wurde (d. h. wenn das Lächeln authentisch war).

Da das wirkliche Lächeln die obere und untere Gesichtshälfte umfasst, wobei die obere Gesichtshälfte das charakteristische „Stirnrunzeln“ signalisiert, das ein Lächeln zu einem echten Lächeln macht, kann es leicht zu Missverständnissen kommen: Gesichtsmasken blockieren das Lächeln und Kommunizieren zugleich das Stirnrunzeln. Das bedeutet, dass ein ernst gemeintes Lächeln aufgrund einer Gesichtsmaske negativer wahrgenommen werden kann als gar kein Lächeln! Da Gesichtsmasken die untere Gesichtshälfte bedecken, ist im Allgemeinen die Fähigkeit, positive Emotionen zu erkennen und zwischen Emotionen zu unterscheiden, erheblich beeinträchtigt. Die Bewegung der Lippen und das Zeigen der Zähne sind für den Betrachter nicht mehr wahrnehmbar, sodass

3 Der französische Neurologe Guillaume Duchenne unterschied in der Mitte des 19. Jahrhunderts als erster die 2 Arten des Lächelns, von denen eines später nach ihm benannt wurde, während er die Physiologie von Gesichtsausdrücken wissenschaftlich untersuchte.

4 Benannt nach der nicht mehr existierenden US-amerikanischen Fluggesellschaft Pan American World Airways, kurz: Pan Am, deren Flugbegleiter darauf trainiert waren, die Fluggäste immer anzulächeln. 
dem Betrachter nur noch die obere Gesichtshälfte zur Erkennung der Emotionen des Maskenträgers zur Verfügung steht.

Ich habe bereits die Möglichkeit erwähnt, nur teilweise offenbarte Gesichtsausdrücke falsch zu interpretieren, d. h. Stirnrunzeln und das Zusammenkneifen der Augenlieder (Blinzeln der Augen) ohne sichtbaren Mund eher als Skepsis, denn als echtes Lächeln misszuverstehen. ${ }^{5}$ Auch können Emotionen wie Überraschung oder Ekel, bei denen es auf die Stellung der Mundwinkel ankommt, als Wut oder Traurigkeit verkannt werden. Gesichtsmasken können mithin sowohl die Wahrnehmung negativer Emotionen verstärken als auch die Wahrnehmung positiver Emotionen beeinträchtigen.

Im Jahr 2017 veröffentlichten Wissenschaftler der Universität Bielefeld Ergebnisse einer experimentellen Studie zur Emotionserkennung, welche die Bedeutung der Augen und des Mundes bei der Wahrnehmung von Emotionen aus Gesichtern sehr deutlich werden ließ. Die Autoren stellten einzelne Gesichter zweier Personen vor, die 6 Grundemotionen (nach Paul Ekman, sowie zusätzlich einen siebten neutralen Gesichtsausdruck) hinter einem 6×8-Raster aus 48 weißen Kacheln zum Ausdruck brachten. Diese wurden nacheinander (in zufälliger Reihenfolge) für die 94 Versuchspersonen aufgedeckt, wobei die Versuchspersonen angewiesen wurden, das Aufdecken zu stoppen, sobald sie die Emotion des Gesichtes erkennen und korrekt benennen können. Für jeden Probanden bestand das Experiment aus 224 Durchgängen (2 Gesichtsidentitäten mal 7 Ausdrücke mal 16 Wiederholungen pro Ausdruck). Mit dieser Methode war es möglich, zu erkennen, welche der 48 Gesichtspartien in welchem Ausmaß dazu beitrugen, dass der Beobachter den aufgedeckten Gesichtsausdruck erkannte. Die Ergebnisse zeigten, dass sich die Beobachter vor allem auf Kacheln stützten, die den Mund und die Augen bedeckten und enthüllten, wenn sie die dargestellte Emotion richtig benannten. Insbesondere identifizierten die Probanden Angst und Trauer vor allem anhand der Augen, wohingegen Ekel und Glück eher beim Fokus auf die Mundregion erkannt wurden [76].

Übertragen auf die Verwendung von Gesichtsmasken im schulischen Umfeld deuten diese Ergebnisse darauf hin, dass Glück und Ekel mit geringerer Wahrscheinlichkeit erkannt werden, während Angst und Traurigkeit mit höherer Wahrscheinlichkeit erkannt werden können. Angesichts der Tatsache, dass die Emotion Ekel im schulischen Umfeld nur selten auftritt, besteht der Nettoeffekt von Gesichtsmasken also darin, dass die Bedeckung des Mundes, d. h. des „Organs des Lächelns“, zu einer verminderten Glückswahrnehmung führt. Darüber hinaus kann das Vorhandensein einer Gesichtsmaske die Motivation des Trägers, überhaupt Mimik zu produzieren, verringern. Da unsere Mimik dem Zweck der Kommunikation dient, wird die Erkenntnis, eine „Kommunikationssperre“ (d. h. Maske) zu tragen, die Bemühungen verringern, Emotionen im Gesicht auszudrücken.

5 Aus diesem Grunde haben plastische Chirurgen empfohlen, Botox-Injektionen um den Musculus orbicularis occuli, wie sie gegen Falten um die Augen herum (Krähenfüße) eingesetzt werden, gegen Stirnrunzeln zu verwenden, um auf diese Weise die Übermittlung falscher emotionaler Signale zu verhindern [59]. Überdies wurde auch schon vorgeschlagen, Oxytocin als Nasenspray dazu zu verwenden, die von den Augen ausgehenden Angstsignale zu dämpfen und Vertrauen zu verstärken, um damit den ungünstigen sozialen Effekten von Gesichtsmasken entgegen zu wirken [40].
Gemäß der „Theorie der verkörperten Emotionen“, die erstmals vor mehr als 100 Jahren von William James vorgeschlagen wurde, kommt es durch einen reduzierten körperlichen Ausdruck von Emotionen (also auch bei reduzierter Mimik) zu einer Verminderung ihres Erlebens. „Sie weinen nicht, weil Sie traurig sind, sondern Sie sind traurig, weil Sie weinen “, haben die Verfechter der Theorie der verkörperten Emotionen plakativ formuliert, um ihre Ansicht, dass wir Emotionen erleben, weil wir bestimmte Verhaltensweisen ausführen (und nicht umgekehrt). Dementsprechend ist man weniger glücklich, sobald man kein Lächeln mehr hervorbringt. Weil zudem die Person, die Emotionen im Gesicht eines Gegenübers aufgrund von dessen Gesichtsmaske nicht wahrnimmt, werden sowohl der Sender als auch der Empfänger des emotionalen Kommunikationssignals von der Gesichtsmaske beeinflusst: Die Emotionalität insgesamt nimmt bei beiden Kommunikationspartnern ab, und dies betrifft die positiven Emotionen mehr als die negativen.

Gleichzeitig wird auch die emotionale Mimikry und damit die emotionale Ansteckung [61] durch die Maske blockiert, d. h. das soziale Phänomen, dass wir unfreiwillig den Gesichtsausdruck anderer Menschen, mit denen wir kommunizieren, nachahmen. Da auch die Emotionen des Zuschauers dessen Gesichtsausdruck folgen, werden seine Emotionen dadurch vermindert. Diese Reaktionen liegen typischerweise außerhalb der bewussten Wahrnehmung und treten innerhalb einer halben Sekunde auf [23].

Zusammenfassend lässt sich sagen, dass das Erkennen und Reagieren auf die Gesichtsausdrücke aller Teilnehmer im Unterricht ein kritischer und notwendiger Bestandteil der sozialen Interaktion in Schulen ist. Sie hilft Schülern und Lehrern, ihr Verhalten zu ändern, um sich an soziale Kommunikations- und Verhaltensnormen anzupassen. Wenn diese emotionalen Signale durch Gesichtsmasken unterdrückt werden, ist unsere Fähigkeit, effektiv miteinander zu kommunizieren, vermindert, und übrig bleibt in erster Linie die Nachahmung negativer (Stirnrunzel-)Emotionen. All dies geschieht von uns unbemerkt und ist daher schwer bewusst zu kontrollieren oder gar zu korrigieren. Da Emotionen eine wichtige Triebkraft für den Gruppenzusammenhalt sind, kann die verminderte Emotionalität, insbesondere die verminderte positive Emotionalität, das reibungslose Geschehen im Klassenzimmer stören. Angesichts der Tatsache, dass jegliches Lernen durch Emotionen erleichtert wird (dies ist ihre hauptsächliche Daseinsberechtigung), ist es wahrscheinlich, dass Gesichtsmasken das Ziel pädagogischen Handelns beeinträchtigen.

\section{Diskussion: Masken oder keine Masken?}

In ihrer Arbeit mit dem an Shakespeare angelehnten Titel („to mask or not to mask") führen Eikenberry und Mitarbeiter abschließend das Folgende an: „Unsere Ergebnisse legen nahe, dass der Gebrauch von Gesichtsmasken möglichst universell (d. h. landesweit) sein und ohne Verzögerung umgesetzt werden sollte, auch wenn die meisten Masken hausgemacht und von relativ geringer Qualität sind. Diese Maßnahme könnte einen wichtigen Beitrag zur Eindämmung der COVID-19-Pandemie leisten, wobei der Nutzen in Verbindung mit anderen nicht pharmazeutischen Interventionen, wie z. B. physischer Distanzierung, die die Übertragung in der Gemeinschaft reduzieren, am größten ist“ [26]. Angesichts dessen, was bisher diskutiert wurde, kann dies kaum das endgültige Verdikt im Hinblick 
auf die positiven und negativen Auswirkungen von Gesichtsmasken im Bildungsbereich während der gegenwärtigen Corona-Pandemie sein.

In einer Gesellschaft, in der die große Mehrheit der Menschen Masken trägt, gibt es viel Raum für gegenseitige emotionale Fehlinterpretationen und damit für Missverständnisse. Menschen können das Gefühl haben, dass jemand ihnen gegenüber aggressiv ist, auch wenn gar keine Aggression vorliegt (sondern tatsächlich sogar ein echtes Lächeln), und entsprechend reagieren - was zu allen möglichen schwierigen und gefährlichen Situationen führen kann. Dies gilt auch für Schulen.

Daher sollten alle Fachkräfte in der Schule über die nachteiligen Auswirkungen von Gesichtsmasken auf die Gesichtserkennung und -identifikation, die Kommunikation und die sozial-emotionale Interaktion Bescheid wissen. Das Tragen von Gesichtsmasken sollten gegen die Alternativen, d. h. Schulschließungen (mit der enormen Belastung für die Kinder und die Eltern) und Wiedereröffnungen von Schulen ohne Masken (mit ihrem erhöhten Risiko von Neuinfektionen) abgewogen werden. In Deutschland sind die Schulbehörden eher zurückhaltend bei der Wiedereröffnung von Schulen nach der Sommerpause, sehen sich aber auch zunehmender Kritik ausgesetzt, weil sie dies nicht tun. Darüber hinaus weisen Lehrergewerkschaften auf das erhöhte Risiko älterer Lehrer hin, an einer potenziell tödlichen Krankheit zu erkranken.

Wie wir in Bildungseinrichtungen mit der Pandemie umgehen und welche Maßnahmen wir treffen, kann nicht unabhängig davon betrachtet werden, was wir ansonsten tun, um die Verbreitung des neuen Coronavirus zu verhindern. Leider ist unser Umgang weit davon entfernt, ausgewogen und widerspruchsfrei zu sein. So müssen wir in Theatern körperlichen Abstand halten, in Flugzeugen aber nicht; wir können mit Masken in Supermärkten einkaufen, aber ohne Masken in Restaurants essen; wir können Versammlungen mit Freunden und Familie abhalten, aber keine Seminare an der Universität usw.

Von besonderer Bedeutung für Schulen können die Erfahrungen mit Gesichtsmasken in Krankenhäusern sein, auch in psychiatrischen Kliniken, z. B. in derjenigen, die ich seit 22 Jahren leite. Am 16. März 2020 habe ich die allgemeine Verwendung von Gesichtsmasken für alle Mitarbeiter und Patienten eingeführt, und es wurde bald klar, dass dies die Ausübung der Psychiatrie behinderte: Die verminderte Beobachtbarkeit emotionaler Veränderungen machten die Arbeit schwieriger. Eine Psychotherapeutin schrieb mir eine E-Mail und bemerkte, vor allem die Psychotherapie mit interaktionell auffälligen/herausfordernden Patienten durch das Tragen der Masken erschwert sei. „Es ist z. B. deutlich schwieriger, dissoziative Zustände oder Anspannung frühzeitig beim Patienten zu erkennen und dann zu intervenieren. Darüber hinaus ist das Durchführen von Expositionsbehandlungen (Trauma-Exposition, Panikstörung, Zwangsstörung etc.), die das bewusste Provozieren von Angst erfordern, mit Maske erschwert. Generell ist der Einsatz von emotionsaktivierenden Verfahren aus der 3. Welle der Verhaltenstherapie mit Masken wahrscheinlich weniger effektiv. Auch in den (Psychotherapie-)Vorgesprächen ist uns aufgefallen, dass das Stellen der Diagnose und die Entscheidung, ob eine Indikation für Psychotherapie gegeben ist, erschwert ist“ [12].

Ein Friseur hatte mir einige Wochen zuvor bereits eine weitere E-Mail gesendet, als der Lockdown gerade vorbei war und er sein
Geschäft wieder aufnehmen konnte: „Ich hatte gestern 2 Neukunden, da muss ich sagen, dass ich sie gebeten hatte, kurzweilig den Mundschutz abzunehmen. Es ist schwierig, zu beraten, wenn man den Kunden nicht vollständig erkennt. Mit meinem Team habe ich sehr unsere Salon-Kommunikation thematisiert, die nun anders abläuft durch den Mundschutz. Man kann Kunden und Befindlichkeiten nicht mehr so gut lesen, wenn die Mimik verdeckt ist. Also achten wir besonders darauf, dass wir positiv kommunizieren, um das fehlende Lächeln auszugleichen “ [72].

Ich bin nicht in der Lage, die Settings Psychotherapie, Friseur oder Schule nach der Bedeutung der emotionalen Bindung in einer Reihe zu ordnen, aber diese Beispiele machen deutlich, dass Schulen keineswegs die einzigen Orte sind, an denen Masken Schwierigkeiten bereiten können. Die geschäftsführende Leiterin der Grundund Hauptschulen (bis zur 10. Klasse) in Ulm sagte mir, dass es viele Diskussionen über das Tragen von Gesichtsmasken gab, als die Corona-Infektionsfälle Mitte März 2020 in Deutschland stark zunahmen. Aber auch in Schulen für geistig behinderte oder verhaltensauffällige Schüler wurden Gesichtsmasken eingeführt und von allen Lehrern und Schülern soweit dies möglich war, getragen. In einigen Schulen wurde körperliche Distanzierung mit oder ohne Maske eingeführt. Einige Lehrer wie auch andere Schulleiter äußerten Bedenken, dass sie sich einen Unterricht mit Gesichtsmasken nicht vorstellen könnten. Die Schulleiterin, mit der ich sprach, äußerte sich dagegen recht positiv über die Möglichkeit, dies in den Schulen zu tun, selbst bei jungen und schwierigen (herausfordernden) Schülern.

Was die Probleme der Kommunikation und der emotionalen Signalisierung betrifft, so ist anzumerken, dass Emotionen auch aus der Körperhaltung entschlüsselt werden können, ein Prozess, der der Entschlüsselung aus Gesichtern [30] sowie aus Prosodie (Sprachmelodie) und Gestik sehr ähnlich ist. Die Behinderung der Kommunikation von Emotionen über das Gesicht kann also bis zu einem gewissen Grad kompensiert werden. Darüber hinaus kann man Emotionen auch direkt ansprechen. Wir sind dies oft nicht gewohnt, gerade weil wir sie permanent ohnehin auf unserem Gesicht vor uns hertragen. Genau so, wie man im psychotherapeutischen Setting lernt, über Emotionen zu sprechen (und sie nicht einfach nur zu haben) können Schüler und Lehrer dies lernen. Die Maßnahmen zur Verringerung der Pandemie sind keine schlechte Gelegenheit, dies zu tun. Hinzu kommt unsere Fähigkeit zum Lernen. Wir wissen aus Erfahrungen mit sehbehinderten oder hörbehinderten Menschen, dass sie lernen, durch Schärfung ihrer intakten Sinne zu kompensieren. Lehrer und Schüler können versuchen, deutlicher zu sprechen, den allgemeinen Lärmpegel im Klassenzimmer gering zu halten und zudem auch genauer hinzuhören. Dann versteht man sich auch mit Masken.

Aus meiner Sicht gilt zusätzlich die folgende allgemeine Überlegung: Gesichtsmasken haben den zusätzlichen Vorteil, dass sie alle in der Schule Anwesenden - Schüler, Lehrer, Verwaltungs- und Hilfspersonal - an die anhaltende Präsenz der Pandemie erinnern und dass ein angemessenes Verhalten für die Sicherheit aller Beteiligten von großer Bedeutung ist. Eine Gesichtsmaske schützt die Person, die sie trägt, und die Person, mit der sie spricht. Trägt diese auch eine Maske, so ist die Chance der Infektion gering ( $\triangleright$ Abb. 1). Die Verwendung einer Maske läuft also darauf hinaus, anderen und sich selbst einen Gefallen zu tun. Sie kann als Paradebeispiel für eine 
kulturelle Norm angesehen werden, die, wenn sie von allen eingehalten wird, der gesamten Gesellschaft erheblichen Nutzen bringt, ohne dass dem Einzelnen dadurch Kosten entstehen. Diese Idee ist allgemein eine der wichtigsten prosozialen Ideen, die traditionell von den obligatorischen Schulsystemen und den sie umsetzenden Staaten gefördert werden. Warum also nicht das Tragen von Gesichtsmasken als einfaches Mittel zur Schulung des Sozialverhaltens einsetzen? Andere solcher „Werkzeuge des Geistes“ wurden weithin vorgeschlagen (Musik, Sport, handwerkliche Kampfkünste), um die Aufmerksamkeit, die exekutive Funktion und die kognitive Kontrolle bei 4- bis 12-Jährigen zu erhöhen [21].

Wenn es schließlich um die Abwägung der Vor- und Nachteile von Gesichtsmasken geht, sind allgemeine und spezifische medizinische und epidemiologische Überlegungen ein wichtiger Teil der Gleichung. Während des Höhepunktes der Pandemie in Deutschland wusste man wenig über Grundlagen wie „wie infektiös sind Kinder/Jugendliche“, „wie wirksam sind Gesichtsmasken wirklich“, „wie ansteckend ist das Virus“ oder „ist das drinnen und draußen gleich?“ - Dies hat sich geändert. Wir wissen z. B., dass Viruspartikel in der Luft im Freien schneller verdünnt werden, empfindlich auf die Umgebungstemperatur und die relative Luftfeuchtigkeit reagieren und durch ultraviolette Strahlung im Sonnenlicht inaktiviert werden. Aus diesem Grund sollten Bildungseinrichtungen im Freien, wo immer möglich, dringend empfohlen werden.

Zur Infektiosität von Kindern und Jugendlichen liegen leider noch immer widersprüchliche Ergebnisse vor. Studien aus Baden-Württemberg und Sachsen legen nahe, dass Schüler bei der Verbreitung von Covid-19 keine große Rolle spielen. Auch internationale Studien legen nahe, dass Kinder sich seltener als andere Menschen infizieren und weniger infektiös sind [20,50]. Andere Studien zeigen dies nicht [73] und zumindest Jugendliche scheinen genauso ansteckend zu sein wie Erwachsene, möglicherweise sogar noch ansteckender. Es besteht die Gefahr, dass junge Menschen das Virus mit nach Hause zu ihren Eltern und Großeltern bringen [10]. Da sie auch mehr tägliche Kontakte haben, sind sie diejenigen, die unbedingt Masken tragen sollten, um andere zu schützen. Zudem gilt: Wenn sogar Kleinkinder in China, Südkorea, Japan und Vietnam dazu in der Lage sind, dann sollten Jugendliche in westlichen Gesellschaften genauso gut dazu in der Lage sein. Auch Hygienevorschriften und entsprechendes Verhalten kann man ihnen spielerisch durchaus beibringen [75].

Schließlich wird die Ausbreitung der Pandemie durch die Zahl der gegenwärtig infizierten Fälle modifiziert. Ich schreibe diesen Aufsatz auf einer Insel in Mecklenburg-Vorpommern, auf der es zum Zeitpunkt des Verfassens dieses Aufsatzes 0 Fälle gab. Es steht also außer Frage, ob hier im Herbst Schulen eröffnet werden sollten. An anderen Orten ist die Lage nicht so ideal. Letztlich gibt es ein Restrisiko, und die vielen Risiken, denen wir im Leben ausgesetzt sind, müssen immer sorgfältig abgewogen werden. Inzwischen planen die Schulen weltweit für den Herbst. Sie müssen dies verantwortungsbewusst tun. Gesichtsmasken sollten Teil des Kalküls sein.
Literatur

[1] Ameres M, Brandstetter S, Tonchev AA, et al. Association of neuronal injury blood marker neurofilament light chain with mild-to-moderate COVID-19. Journal of Neurology 2020; doi.org/10.1007/s00415-02010050-y

[2] Anfinrud P, Stadnytskyi V, Bax CE, et al. Visualizing speech-generated oral fluid droplets with laser light scattering. N Engl J Med 2020; 382: 2061-2063

[3] Anonymus. Generation Coronavirus? The Lancet 2020; 395: 1949

[4] Anonymus. Physical distancing, face masks, and eye protection for prevention of covid-19. The Lancet 2020; 395: 1950-1951

[5] Asadi S, Wexler AS, Cappa CD, et al. Aerosol emission and superemission during human speech increase with voice loudness. Sci Rep 2019; 9: 2348

[6] Asadi S, Bouvier N, Wexler AS, et al. The coronavirus pandemic and aerosols: Does COVID-19 transmit via expiratory particles?. Aerosol Sci Technol 2020. doi:10.1080/02786826.2020.1749229

[7] Ashcraft MH. Math anxiety and its cognitive consequences. Curr Dir Psychol Sci 2020; 11: 181-185

[8] Atcherson SR, Mendel LL, Baltimore W], et al. The Effect of Conventional and Transparent Surgical Masks on Speech Understanding in Individuals with and without Hearing Loss. J Am Acad Audiol 2017; 28: 58-67

[9] Bai N. Still Confused About Masks? Here's the Science Behind How Face Masks Prevent Coronavirus. University of California San Francisco (UCSF) Special Notice Concerning Covid-19, June 26th 2020. https:// www.ucsf.edu/news/2020/06/417906/still-confused-about-masks-heres-science-behind-how-face-masks-prevent; accessed July 11th 2020

[10] Bernstein L. Young people are infecting older family members in shared homes. The Washington Post July 30th, 2020. https://www. washingtonpost.com/health/young-people-are-infe...5aCZ3cG1rPTEifQ.O-CuALMWh27tdFtRbky1nubiWgW8ysvLDpJiRWpObSg; accessed 4.8.2020

[11] Bombari D, Schmid PC, Schmid Mast M, et al. Emotion recognition: the role of featural and configural face information. Q J Exp Psychol 2013; 66: 2426-2442

[12] Bosch J. Persönliche Mitteilung via E-Mail vom 18.6.2020

[13] Brann DH, Tsukahara T, Weinreb C, et al. Non-neuronal expression of SARS-CoV-2 entry genes in the olfactory system suggests mechanisms underlying COVID-19-associated anosmia. Sci Adv 2020. 10.1126/ sciadv.abc5801

[14] Chu DK, Akl EA, Duda S, et al. Physical distancing, face masks, and eye protection to prevent person-to-person transmission of SARS-CoV-2 and COVID-19: a systematic review and meta-analysis. Lancet 2020; 395: 1973-1987

[15] Cohn S. Face masks: what the Spanish flu can teach us about making them compulsory. The Conversation May 1st, 2020. https://theconversation.com/face-masks-what-the-spanish-flu-can-teach-us-about-making-them-compulsory-137648; accessed July 14th 2020

[16] Couzin-Frankel J, Vogel G, Weiland M. Not open and shut. School openings across the globe suggest ways to keep the coronavirus at bay, despite outbreaks. Science 2020; 369: 241-245

[17] COVID-19 Dashboard. COVID-19 Dashboard by the Center for Systems Science and Engineering (CSSE) at Johns Hopkins University (JHU). https://www.arcgis.com/apps/opsdashboard/index.html\#/bda7594740fd40299423467b48e9ecf6; accessed July 29th 2020

[18] Curby KM, Johnson KJ, Tyson A. Face to face with emotion: Holistic face processing is modulated by emotional state. Cognition and Emotion 2012; 26: 93-102

[19] Darwin C. The Expression of the Emotions in Man and Animals. London: Fontana Press; 1872, 1999 
[20] Davies, NG Klepac P, Liu Y, et al. Age-dependent effects in the transmission and control of COVID-19 epidemics. Mature medicine 2020. doi.org/10.1038/s41591-020-0962-9

[21] Diamond A, Barnett WS, Thomas ], et al. Preschool Program Improves Cognitive Control. Science 2007; 318: 1387-1388

[22] Diamond A, Lee K. Interventions Shown to Aid Executive Function Development in Children 4 to 12 Years Old. Science 2011; 333: 959-964

[23] Dimberg U, Thunberg M, Elmehed K. Unconscious facial reacions to emotional facial expressions. Psychological Science 2000; 11: 86-89

[24] Duguid JP. The size and the duration of air-carriage of respiratory droplets and droplet-nuclei. J Hyg 1946; 44: 471-479

[25] Eibl-Eibesfeld I. Grundriß der vergleichenden Verhaltensforschung. München: Piper, 1975

[26] Eikenberry SE, Mancuso M, Iboi E, et al. To mask or not to mask: Modeling the potential for face mask use by the general public to curtail the COVID-19 pandemic. Infectious Disease Modelling 2020; 5: 293-308

[27] Ekman P. Universal facial expressions of emotion. California Mental Health Research Digest 1970; 8: 151-158

[28] Ekman P, Friesen WV. Constants across cultures in the face and emotion. J Pers Soc Psychol 1971; 17: 124-129

[29] Ekman P. Are there basic emotions? Psychol Rev 1992; 99: 550-553

[30] Enea V, lancu S. Processing emotional body expressions: state-of-theart. Soc Neurosci 2016; 11: 495-506

[31] Esposito S, Principi N, Leung CC, et al. Universal use of face masks for success against COVID-19: evidence and implications for prevention policies. Eur Respir J 2020. doi.org/10.1183/13993003.01260-2020

[32] Fikenzer S, Uhe T, Lavall D, et al. Effects of surgical and FFP2/N95 face masks on cardiopulmonary exercise capacity. Clinical Research in Cardiology 2020. doi.org/10.1007/s00392-020-01704-y

[33] Fox SE, Akmatbekov A, Harbert JL, et al. Pulmonary and cardiac pathology in African American patients with COVID-19: an autopsy series from New Orleans. Lancet Respir Med 2020. doi.org/10.1016/S22132600(20)30243-5

[34] Götzinger F, Santiago-García B, Noguera-Julián A, et al. COVID-19 in children and adolescents in Europe: a multinational, multicentre cohort study. Lancet Child Adolesc Health 2020. doi.org/10.1016/ S2352-4642(20)30177-2; accessed 26th June 2020

[35] Gunnery SD, Ruben MA. Perceptions of Duchenne and non-Duchenne smiles: A meta-analysis. Cognition and Emotion 2016; 30: 501-515

[36] Henrich J. The secret of our success. Princeton: University Press, 2016

[37] Hoehl S, Striano T. Neural processing of eye gaze and threat-related emotional facial expressions in infancy. Child Development 2008; 79: $1752-1760$

[38] Jack RE, Schyns PG. The Human Face as a Dynamic Tool for Social Communication. Current biology 2015; 25: R621-R634

[39] Johnson KJ, Waugh CE, Fredrickson BL. Smile to see the forest: Facially expressed positive emotions broaden cognition. Cognition and Emotion 2010; $24: 299-321$

[40] Kanat M, Heinrichs M, Mader I, et al. Oxytocin Modulates Amygdala Reactivity to Masked Fearful Eyes. Neuropsychopharmacology 2015; 40: $2632-2638$

[41] Krys K, Vauclair C, Capaldi CA, et al. Be Careful Where You Smile: Culture Shapes Judgments of Intelligence and Honesty of Smiling Individuals. J Nonverbal Behav 2016; 40: 101-116

[42] Lavezzo E, Franchin E, Ciavarella C, et al. Suppression of a SARS-CoV-2 outbreak in the Italian municipality of Vó. Nature2020. doi:10.1038/ s41586-020-2488-1

[43] Lawton G. You could be spreading the coronavirus without realising you've got it. New Scientist 2020; 3275

[44] Lazzarino Al, Steptoe A, Hamer M, et al. Covid-19: Important potential side effects of wearing face masks that we should bear in mind. BM] 2020; 369: m2003
[45] Leung NHL, Chu DKW, Shiu EYC, et al. Respiratory virus shedding in exhaled breath and efficacy of face masks. Nature Medicine 2020; 26 : 676-680

[46] Li Y, Tokura H, Guo YP, et al. Effects of wearing N95 and surgical facemasks on heart rate, thermal stress and subjective sensations. Int Arch Occup Environ Health 2005; 78: 501-509

[47] Li R, Pei S, Chen B, et al. Substantial undocumented infection facilitates the rapid dissemination of novel coronavirus (SARS-CoV-2). Science2020; 368: 489-493

[48] Libertus K, Landa RJ, Haworth JL. Development of Attention to Faces during the First 3 Years: Influences of Stimulus Type. Front Psychol 2017; 8: 1976

[49] Liu Y, Ning Z, Chen Y, et al. Aerodynamic analysis of SARS-CoV-2 in two Wuhan hospitals. Nature 2020; 582: 557-560

[50] Ludvigsson JF. Children are unlikely to be the main drivers of the COVID-19 pandemic - A systematic review. Acta Paediatr 2020; 109: $1525-1530$

[51] Lyons IM, Beilock SL. Mathematics Anxiety: Separating the Math from the Anxiety. Cerebral Cortex 2012; 22: 2102-2110

[52] Lyu W, Wehby GL. Community Use Of Face Masks And COVID-19: Evidence From A Natural Experiment Of State Mandates In The US. Health Affairs, published online ahead of print June 16th, 2020. https:// www.healthaffairs.org/doi/10.1377/hlthaff.2020.00818?cookieSet=1; accessed June 20th 2020

[53] Maclntyre CR, Wang Q. Physical distancing, face masks, and eye protection for prevention of Covid-19. Lancet 2020; 395: 1959-1951

[54] Mendel LL, Gardino JA, Atcherson SR. Speech understanding using surgical masks: a problem in health care?. J Am Acad Audiol 2002; 19: 686-695

[55] Meselson M. Droplets and Aerosols in the Transmission of SARS-CoV-2. N Engl J Med 2020; 382: 2063

[56] Mitze T, Kosfeld R, Rode ], et al. [Compulsory face masks and their effects on the corona pancemic. What the world may learn from Jena; original title in German]. Working paper from June 3 rd 2020, University of Southern Denmark, University of Kassel, Technical University Darmstadt, Johannes Gutenberg University Mainz. https://download. uni-mainz.de/presse/03_wiwi_corona_masken_paper_zusammenfassung.pdf; accessed June 9th 2020

[57] Moustafa AA, Tindle R, Ansari Z, et al. Mathematics, anxiety, and the brain. Reviews in the Neurosciences 2017; 28. doi.org/10.1515/revneuro-2016-0065

[58] Müller-Pinzler L, Krach S, Krämer UM, et al. The Social Neuroscience of Interpersonal Emotions. Social Behavior from Rodents to Humans 2016: 241-256

[59] Nestor MS, Fischer D, Arnold D. “Masking” our Emotions: Botulinum Toxin, Facial Expression and Well-Being in the Age of COVID-19. Journal of Cosmetic Dermatology/Early View, first published June 27th 2020. doi.org/10.1111/jocd.13569; accessed July 7th, 2020

[60] Ong JJY, Bharatendu C, Goh Y, et al. Headaches Associated With Personal Protective Equipment - A Cross-Sectional Study Among Frontline Healthcare Workers During COVID-19. Headache 2020; 60: 864-877

[61] Olszanowski M, Wróbel M, Hess U. Mimicking and sharing emotions: a re-examination of the link between facial mimicry and emotional contagion. Cognition and Emotion 2020; 34: 367-376

[62] Oxley T], Mocco J, Majidi S, et al. Large-Vessel Stroke as a Presenting Feature of Covid-19 in the Young. NEJM 2020. doi: 10.1056/NEJMc2009787

[63] Pascalis O, Scott LS, Kelly DJ, et al. Plasticity of face processing in infancy. PNAS 2005; 102: 5297-5300

[64] Peltola MJ, Leppänen JM, Mäki S, et al. Emergence of enhanced attention to fearful faces between 5 and 7 months of age. Social Cognitive and Affective Neuroscience 2019; 4: 134-142 
[65] Phelamei S. Wearing face masks is the "new Normal": Know these tips and tricks to avoid common side effects. The Times Now, June 10th, 2020

[66] Pons F, Bosch L, Lewkowicz DJ. Bilingualism Modulates Infants' Selective Attention to the Mouth of a Talking Face. Psychological Science 2015; 26: 490-498

[67] Prather KA, Wang CC, Schooley RT et al. Reducing transmission of SARSCoV-2. Masks and testing are necessary to combat asymptomatic spread in aerosols and droplets. Science 2020; 368: 1422-1424

[68] Rapkiewicz AV, Mai X, Carsons SE, et al. Megakaryocytes and platelet-fibrin thrombi characterize multi-organ thrombosis at autopsy in COVID-19: A case series. EClinicalMedicine 2020. doi.org/10.1016/j. eclinm.2020.100434

[69] Reynolds GD, Roth KC. The Development of Attentional Biases for Faces in Infancy: A Developmental Systems Perspective. Front Psychol 2018; 9: 222

[70] Rivett L, Sridhar S, Sparkes D, et al. Screening of healthcare workers for SARS-CoV-2 highlights the role of asymptomatic carriage in COVID-19 transmission. Elife 2020; 9: e58728

[71] Scheller E, Büchel C, Gamer M. Diagnostic Features of Emotional Expressions Are Processed Preferentially. PLoS ONE 2012; 7: e41792

[72] Spitzer M. Pandemie. Was die Krise mit uns macht und was wir aus ihr machen. München: Münchner Verlagsgruppe (mvg), 2020

[73] Szablewski CM, Chang KT, Brown MM, et al. SARS-CoV-2 Transmission and Infection Among Attendees of an Overnight Camp - Georgia, June 2020. Centers for Disease Control and Prevention MMWR 2020; 69, dx.doi.org/10.15585/mmwr.mm6931e1external icon

[74] UNESCO. Education: From disruption to recovery. School clusures caused by Coronavirus (Covid-19). https://en.unesco.org/covid19/ educationresponse, accessed July 11th 2020

[75] Walger $P$, Heininger $U$, Knuf M, et al. Children and adolescents in the CoVid-19 pandemic: Schools and daycare centers are to be opened again without restrictions. The protection of teachers, educators, carers and parents and the general hygiene rules do not conflict with this. GMS Hyg Infect Control 2020; 15: Doc11

[76] Wegrzyn M, Vogt M, Kireclioglu B, et al. Mapping the emotional face. How individual face parts contribute to successful emotion recognition. PLoS One 2017; 12: e0177239

[77] Wößmann L. On the economic costs of not-learning. What we know about school-lockdowns from educational research (Folgekosten ausbleibenden Lernens: Was wir über die Corona-bedingten SchulschlieBungen aus der Forschung lernen können.) Ifo Schnelldienst 2020; 73, June 10th 2020 